\title{
Renal safety of tenofovir and/or entecavir in patients with chronic HBV monoinfection
}

\author{
This article was published in the following Dove Press journal: \\ Therapeutics and Clinical Risk Management \\ 26 September 2017 \\ Number of times this article has been viewed
}

\section{Young-Mo Yang \\ Eun Joo Choi}

Department of Pharmacy, College of Pharmacy, Chosun University, Gwangju, South Korea
Correspondence: Eun Joo Choi

Department of Pharmacy, College

of Pharmacy, Chosun University,

309 Pilmun-daero, Dong-gu,

Gwangju 61452, South Korea

Tel +82622306382

Fax +82 622225414

Email ejchoi@chosun.ac.kr
Background: Tenofovir disoproxil fumarate (TDF) and entecavir (ETV) are recommended as the first-line therapy for chronic hepatitis B (CHB) due to their genetic barrier to resistance and effectiveness of virological suppression. TDF and ETV may cause renal toxicity through various mechanisms such as renal tubular injury, apoptosis, and mitochondrial toxicity. The aims of the current review were to assess the potential renal toxicity associated with the use of TDF and ETV in patients infected with chronic hepatitis B virus (HBV) and to provide clinical perspectives on these two agents in the treatment of CHB.

Methods: A literature search of clinical studies published in PubMed and posted on ClinicalTrials.gov website was implemented to find studies evaluating the potential renal toxicity of TDF and ETV.

Results: Twenty-one studies were examined in this review. The TDF dose used in the studies was 245 or $300 \mathrm{mg}$ /day and that of ETV was 0.5 or $1 \mathrm{mg} /$ day. Based on the markers of renal function, patients treated with TDF were not more likely to show changes in renal function than those treated with ETV; however, the estimated glomerular filtration rates (eGFRs) of patients receiving TDF tended to be more clearly reduced than those of patients receiving ETV. The eGFRs of patients treated with TDF decreased in a time-dependent manner, whereas those of patients treated with ETV increased or decreased across various time points.

Conclusion: The data shown in this study suggest that use of TDF and ETV could be at least associated with reductions in renal function in patients with chronic HBV infection. However, various risk factors, such as pre-existing renal failure and comorbidities, are also associated with decreased renal function during the treatment of TDF and ETV. Thus, studies of management strategies for HBV-infected patients with these risk factors are necessary in the near future.

Keywords: hepatitis B, tenofovir, entecavir, renal safety

\section{Introduction}

Hepatitis B virus (HBV) infection is considered as one of the most important global public health concerns; this potentially life-threatening infection damages the liver and can contribute to acute and chronic diseases. An estimated 240 million individuals are chronically infected with HBV worldwide, and over 686,000 individuals die annually because of end-stage chronic hepatitis $\mathrm{B}$ (CHB) and CHB-associated complications such as decompensated cirrhosis and hepatocellular carcinoma. ${ }^{1}$

Currently, two therapeutic options (ie, interferons [IFNs] and oral nucleos(t)ide analogs [NUCs]) are used to treat CHB; however, oral NUCs have been preferred for the treatment of CHB owing to their convenient regimen. ${ }^{2}$ In particular, the secondgeneration NUCs, such as entecavir (ETV) and tenofovir disoproxil fumarate (TDF), are recommended as the first-line therapy for $\mathrm{CHB}$ because of their high genetic barrier to resistance and effectiveness of virological suppression. ${ }^{3,4}$ The efficacy and safety 
of both these drugs were demonstrated through previous clinical trials. ${ }^{3}$ Safety should be particularly considered, since long-term treatment for CHB is usually required with ETV or TDF, although its ideal duration of treatment is not well determined. ${ }^{5}$

ETV and TDF may cause renal toxicity via various mechanisms such as renal tubular injury, apoptosis, and mitochondrial toxicity. ${ }^{5,6}$ Previous studies also reported an association between $\mathrm{CHB}$ and chronic kidney disease (CKD). ${ }^{7-11}$ Specifically, it was reported that glomerular diseases, such as membranous nephropathy and mesangiocapillary glomerulonephritis, might be the underlying causes of renal dysfunction in patients with CHB. ${ }^{12,13}$ Moreover, drug history except for NUCs, disease status of diabetes and/or hypertension (HTN), and baseline (BL) kidney function before starting NUCs may affect the potential nephrotoxicity caused by ETV and/or TDF. Consequently, renal safety is an important factor in choosing appropriate NUCs for the treatment of CHB because they are renally eliminated in an unchanged form, and this is particularly important in patients who have already had renal impairment or are at risk for it. ${ }^{7,14}$

The current review aimed to assess the potential renal toxicity associated with the use of ETV and TDF in patients infected with chronic HBV and to provide clinical perspectives on these two agents in the treatment of CHB.

\section{Methods}

A literature search was conducted to identify clinical studies in patients with HBV monoinfection, which assessed the safety of ETV and/or TDF. PubMed was searched from the inception of the database to March 2017, using "hepatitis B," "entecavir," and "tenofovir" as the search terms to find clinical trials written only in English. The reference lists of the selected articles and related reviews were utilized to find additional relevant articles. The data posted on ClinicalTrials.gov website were also used to identify the unpublished clinical outcomes. Two reviewers independently scanned the article titles and abstracts and identified relevant studies that met the following criteria: 1) retrospective or prospective clinical studies, 2) studies involving patients only with HBV infection, 3) studies in which ETV and/or TDF had to be administered for the treatment of HBV infection, and 4) studies whose results contained renal parameters, such as estimated glomerular filtration rate (eGFR), serum creatinine, and serum phosphorus, in order to evaluate the changes in renal function.

\section{Results \\ Study characteristics}

The literature search (Figure 1) identified 21 eligible studies that met the predetermined inclusion criteria. The main characteristics of the selected studies are presented in Table 1.

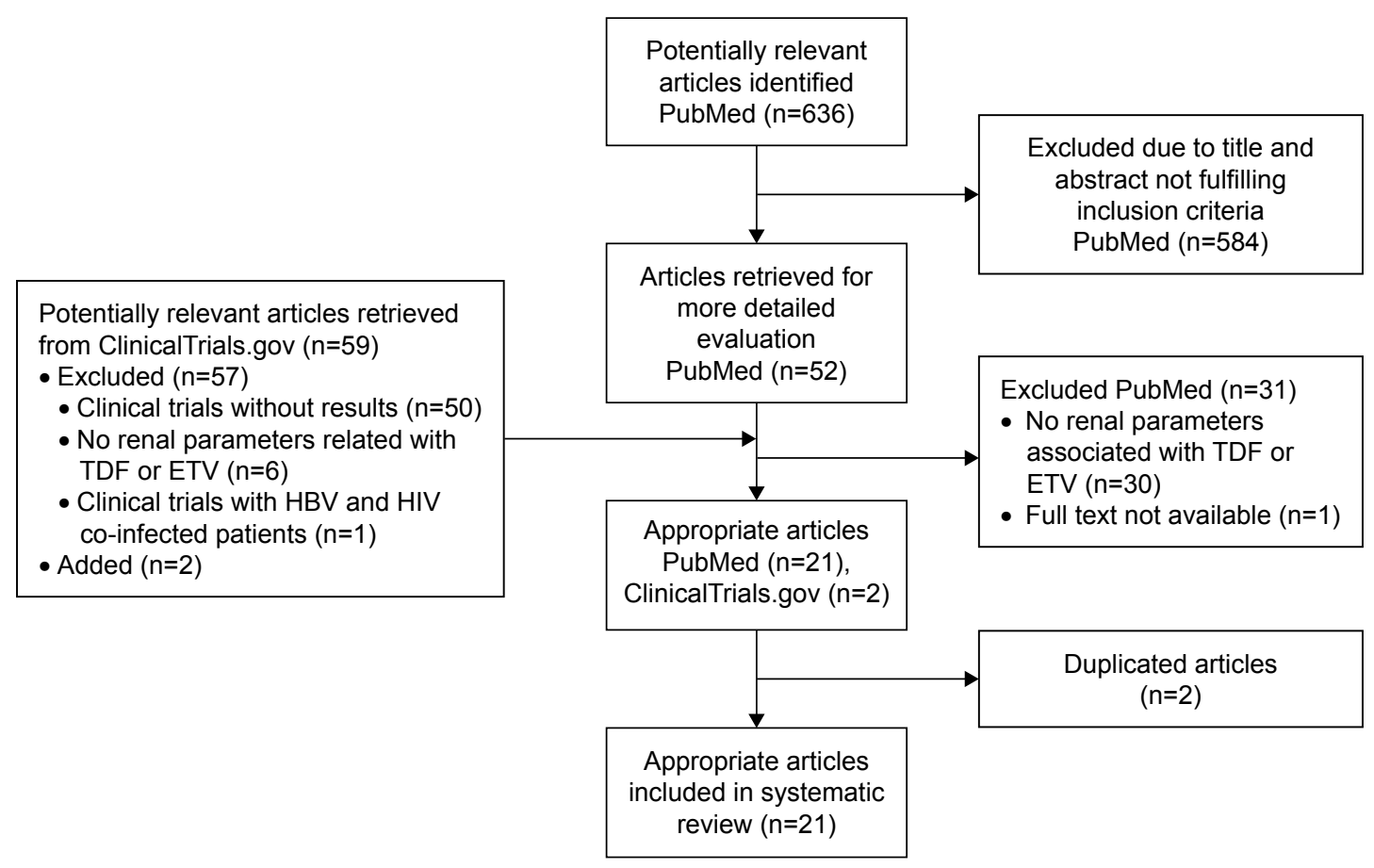

Figure I Flowchart of the study selection process.

Abbreviations: ETV, entecavir; HBV, hepatitis B virus; TDF, tenofovir disoproxil fumarate. 


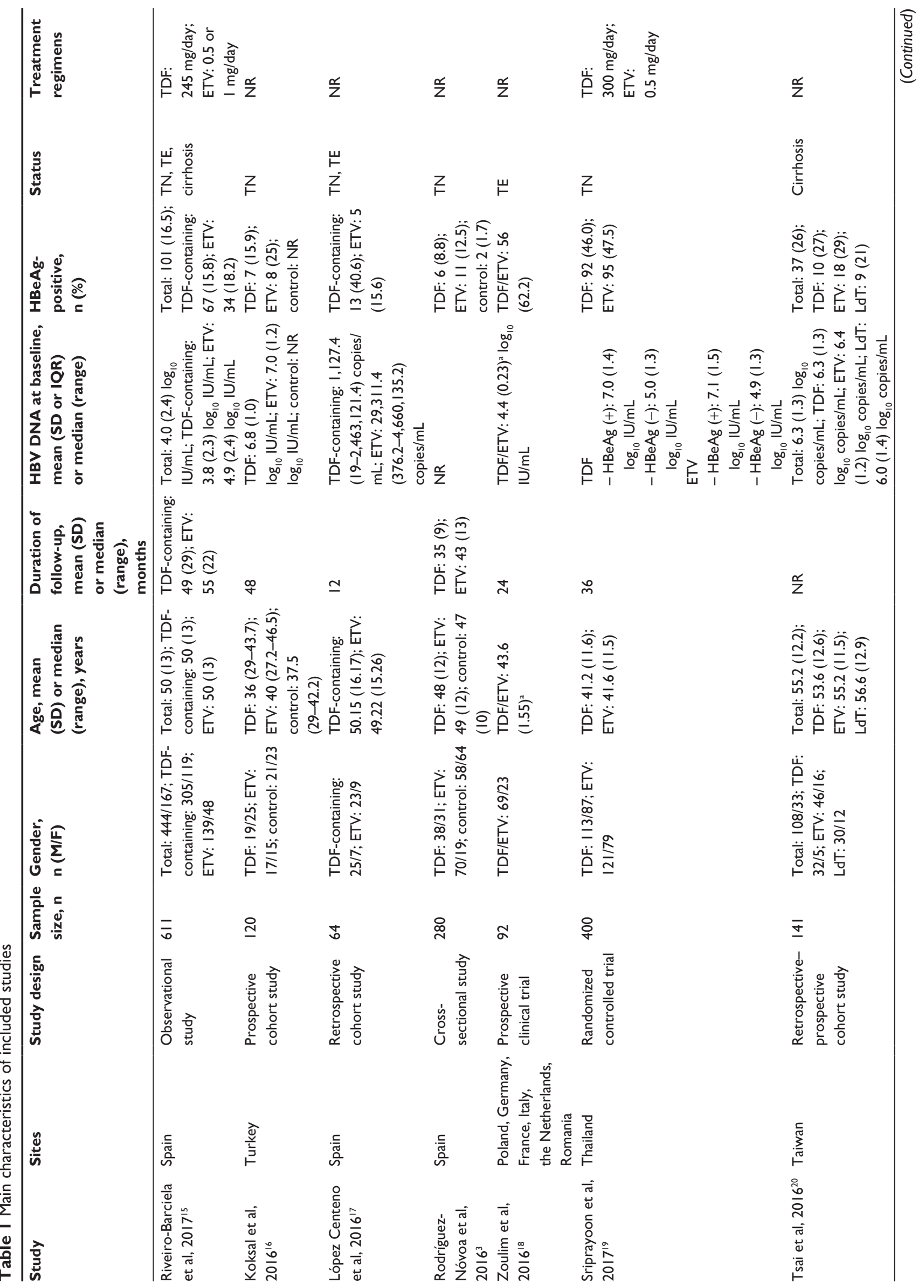




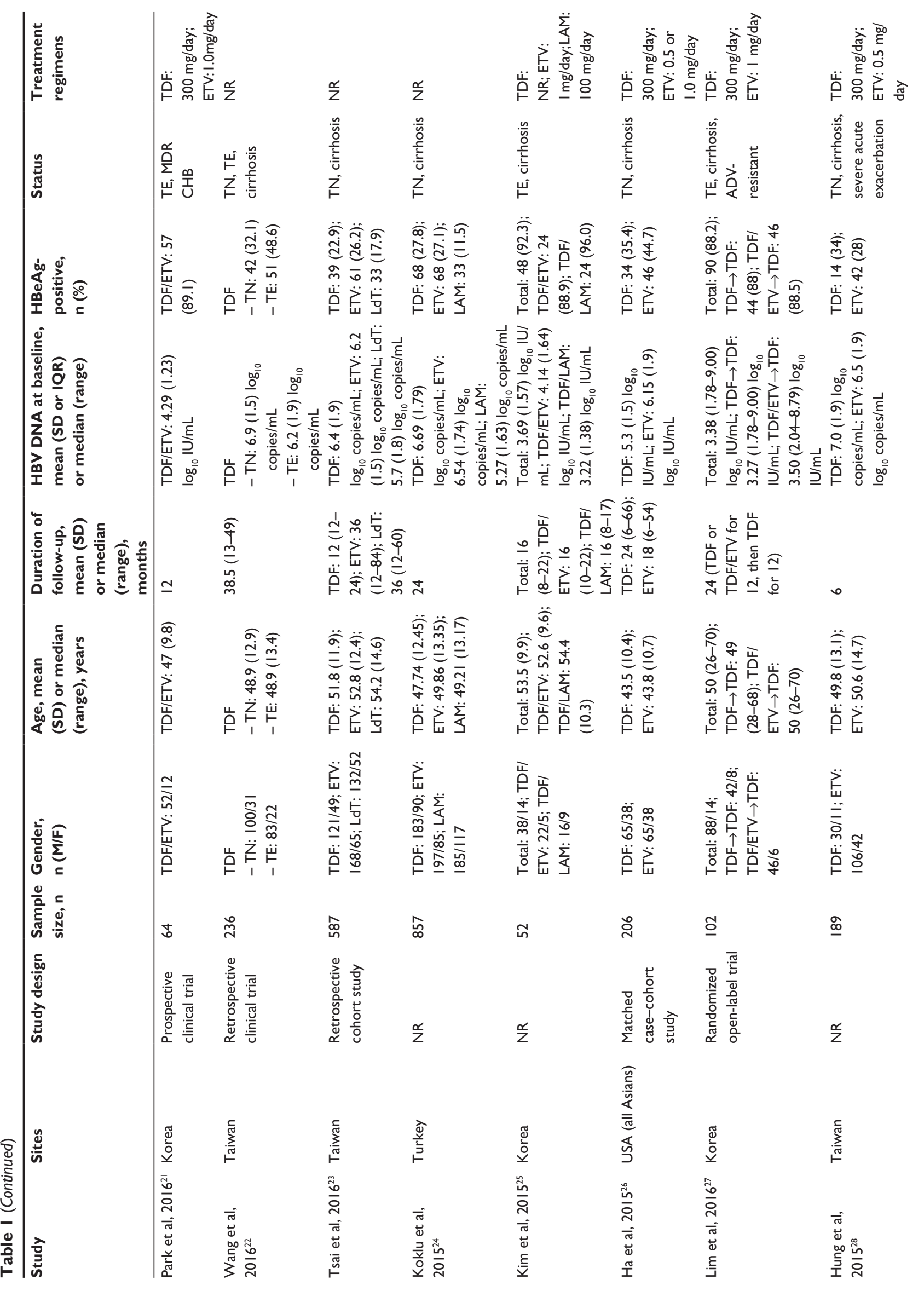




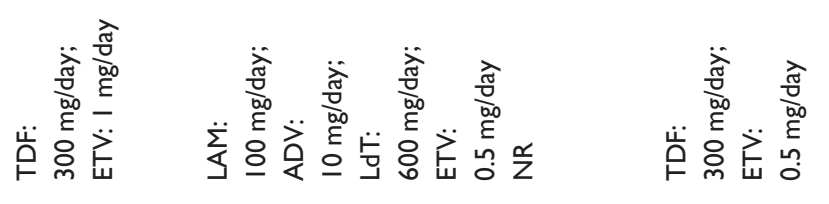

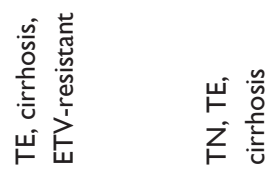

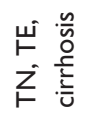

$z$

言芦

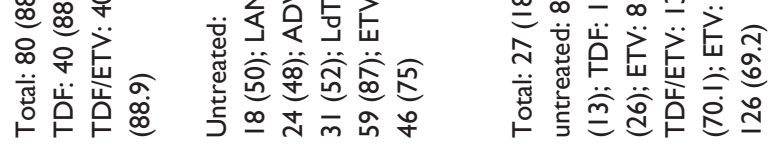

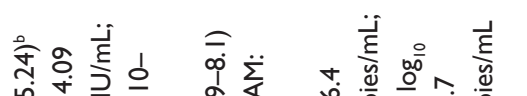

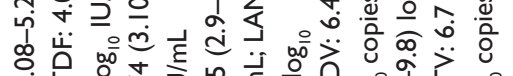

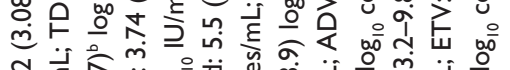

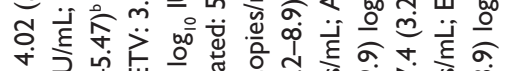

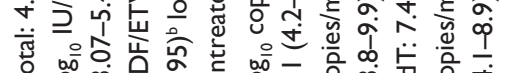

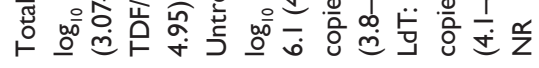

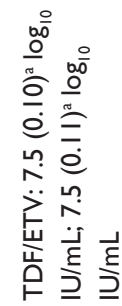

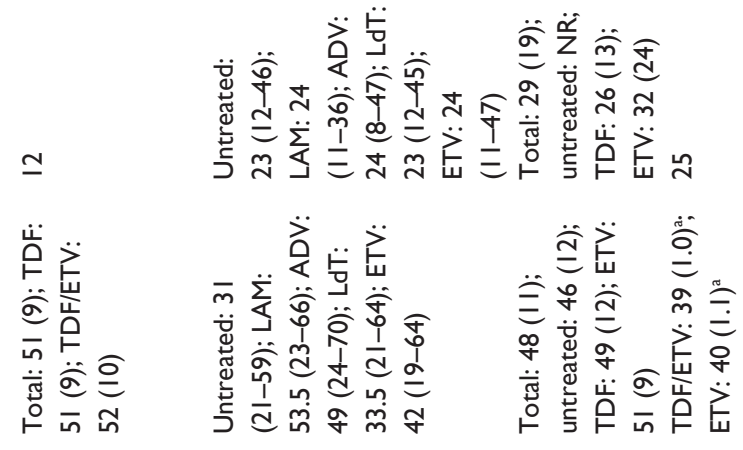

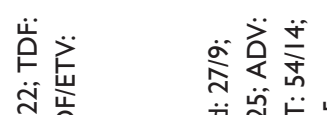

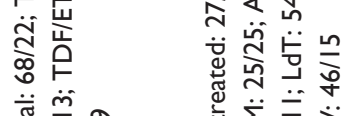

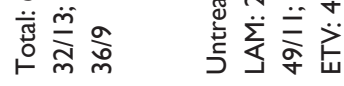

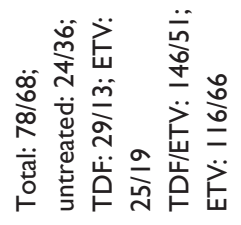

$\stackrel{\infty}{z}$
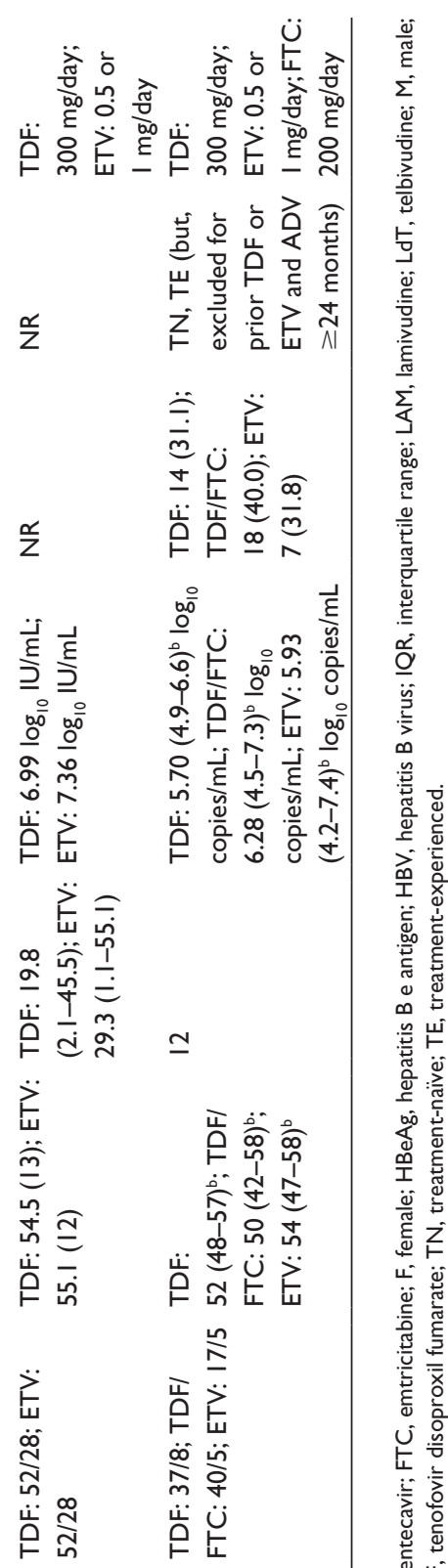

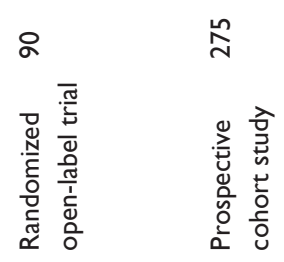

I

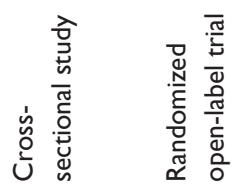

$\stackrel{x}{z}$

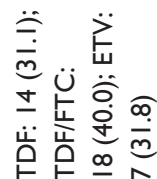

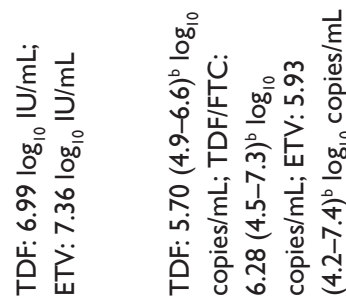

竞焉

岗突

苨

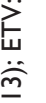

นก้ำ

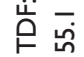

$\simeq$

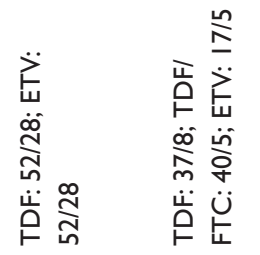

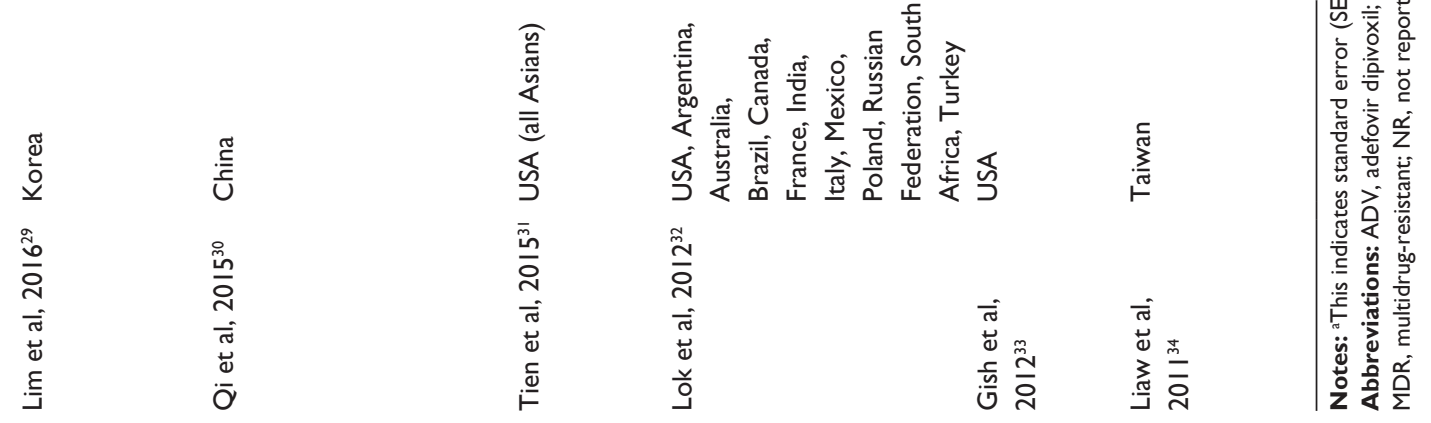


The final eligible studies included in this review were conducted in the United States, Europe, Thailand, Taiwan, Korea, and China. ${ }^{3,15-34}$ In particular, 13 studies were conducted on Asian individuals. ${ }^{19-23,25-31,34}$ Most of the studies (61.9\%), excluding five randomized clinical studies ${ }^{19,27,29,32,34}$ and three studies that did not accurately report study designs, ${ }^{24,25,28}$ were observational studies. Most studies were published in the last 2 years, although the articles dated back to 2011. Overall, $95.2 \%(20 / 21)$ of the studies were conducted on patients with mixed hepatitis $\mathrm{B}$ e antigen ( $\mathrm{HBeAg}$ ) status whereas only one study ${ }^{33}$ did not report HBeAg status. The TDF dose used in the studies was 245 or $300 \mathrm{mg} /$ day and that of ETV was 0.5 or $1 \mathrm{mg} /$ day.

\section{Evaluation of renal safety of TDF and ETV}

Information bias that may result from broad heterogeneity in the methodology among different studies was the major issue hindering meta-analyses. As presented in Table 2, various parameters were utilized in order to measure renal functions after administering TDF and ETV. The most common parameter used was eGFR calculated using modification of diet in renal disease (MDRD) and CockcroftGault (CG) formulae.

An observational study comparing long-term renal functions reported eGFR in 424 patients with TDF-containing regimens and 187 patients with ETV according to the MDRD method. ${ }^{15}$ In the patients with TDF-containing regimens, the mean eGFR decreased from $90.8 \mathrm{~mL} / \mathrm{min}$ at $\mathrm{BL}$ to $85.1 \mathrm{~mL} / \mathrm{min}$ at 60 months. However, in the patients with ETV, the mean eGFR increased from $81.2 \mathrm{~mL} / \mathrm{min}$ at $\mathrm{BL}$ to $90.7 \mathrm{~mL} / \mathrm{min}$ at 60 months. A retrospective-prospective cohort study conducted in Taiwan determined a change in eGFR in 37 patients with TDF and 62 patients with ETV based on the MDRD method. ${ }^{20}$ In the patients with TDF, the mean eGFR changed from $78.3 \mathrm{~mL} / \mathrm{min} / 1.73 \mathrm{~m}^{2}$ at $\mathrm{BL}$ to $73.0 \mathrm{~mL} / \mathrm{min} / 1.73 \mathrm{~m}^{2}$ at 24 months, whereas in the patients with ETV, the mean eGFR increased from $75.6 \mathrm{~mL} / \mathrm{min} / 1.73 \mathrm{~m}^{2}$ at $\mathrm{BL}$ to $79.3 \mathrm{~mL} / \mathrm{min} / 1.73 \mathrm{~m}^{2}$ at 24 months. A similar change in eGFR calculated using the MDRD method was also observed in a retrospective cohort study conducted in Taiwan. ${ }^{23}$ The mean eGFR in 170 patients with TDF changed from $92 \mathrm{~mL} / \mathrm{min} / 1.73 \mathrm{~m}^{2}$ at BL to 86.3 $\mathrm{mL} / \mathrm{min} / 1.73 \mathrm{~m}^{2}$ at 24 months; however, in 233 patients with ETV, the mean eGFR changed from $86.1 \mathrm{~mL} / \mathrm{min} / 1.73 \mathrm{~m}^{2}$ at BL to $94.4 \mathrm{~mL} / \mathrm{min} / 1.73 \mathrm{~m}^{2}$ at 24 months.

A prospective cohort study conducted in Turkey reported a change in eGFR in 44 patients with TDF and 32 patients with ETV according to the Chronic Kidney Disease Epidemiology
Collaboration and cystatin C (CKD-EPI-CysC) method. ${ }^{16}$ The mean eGFR in the patients with TDF decreased from $84.7 \mathrm{~mL} /$ $\mathrm{min} / 1.73 \mathrm{~m}^{2}$ at BL to $76.9 \mathrm{~mL} / \mathrm{min} / 1.73 \mathrm{~m}^{2}$ at 24 months $(p=0.004)$ and that in the patients with ETV decreased from $90.0 \mathrm{~mL} / \mathrm{min} / 1.73 \mathrm{~m}^{2}$ at BL to $84.5 \mathrm{~mL} / \mathrm{min} / 1.73 \mathrm{~m}^{2}$ at 24 months $(p=0.46)$. However, the mean values of eGFR in both groups were different when the Chronic Kidney Disease Epidemiology Collaboration and creatinine plus cystatin $\mathrm{C}$ (CKD-EPI-Cr-CysC) method was used. The mean eGFR in the patients with TDF changed from $90.6 \mathrm{~mL} / \mathrm{min} / 1.73 \mathrm{~m}^{2}$ at $\mathrm{BL}$ to $73.6 \mathrm{~mL} / \mathrm{min} / 1.73 \mathrm{~m}^{2}$ at 24 months $(p=0.05)$, and the mean eGFR in the patients with ETV changed from $93.5 \mathrm{~mL} / \mathrm{min} / 1.73 \mathrm{~m}^{2}$ at $\mathrm{BL}$ to $82.3 \mathrm{~mL} / \mathrm{min} / 1.73 \mathrm{~m}^{2}$ at 24 months $(p=0.17)$. A study conducted by Koklu et $\mathrm{al}^{24}$ in Turkey reported eGFR calculated using the MDRD method. The mean eGFR in 273 patients with TDF changed from $100.72 \mathrm{~mL} / \mathrm{min} / 1.73 \mathrm{~m}^{2}$ at BL to $96.72 \mathrm{~mL} / \mathrm{min} / 1.73 \mathrm{~m}^{2}$ at 24 months ( $p=0.001)$, whereas the mean eGFR in 282 patients with ETV changed from $96.20 \mathrm{~mL} / \mathrm{min} / 1.73 \mathrm{~m}^{2}$ at $\mathrm{BL}$ to $95.94 \mathrm{~mL} / \mathrm{min} / 1.73 \mathrm{~m}^{2}$ at 24 months $(p=0.535)$. In a study conducted by Hung et $\mathrm{al}^{28}$ in Taiwan, TDF and ETV showed decreased mean eGFR calculated using the MDRD method. The mean eGFR in 41 patients with TDF changed from 108 to $87 \mathrm{~mL} / \mathrm{min} / 1.73 \mathrm{~m}^{2}$ at 6 months $(p=0.001)$, and the mean eGFR in 148 patients with ETV changed from 92 to $84 \mathrm{~mL} / \mathrm{min} / 1.73 \mathrm{~m}^{2}$ at 6 months $(p=0.001)$.

\section{Discussion}

Close attention should be paid to the safety and efficacy of TDV and ETV for the long-term treatment of chronic HBV infection, because they are currently the most potent antiviral agents for treating HBV infection. ${ }^{3,5}$ TDF and ETV are likely to cause renal toxicity through various mechanisms including renal tubular injury, apoptosis, and mitochondrial toxicity., The present study reviewed the literature and provided a comprehensive summary of the renal safety of TDF and ETV for the treatment of patients with chronic HBV infection. The results based on the studies reviewed in this article indicated that TDF and ETV could be responsible at least for reduced kidney function in patients with chronic HBV infection.

In this study, the effects of TDF and ETV on renal function were assessed. Based on the markers of renal function, compared to patients treated with ETV, those treated with TDF were not more likely to show changes in renal function, although the eGFR of patients treated with TDF tended to be more clearly reduced than that of patients receiving ETV. The eGFRs of patients treated with TDF decreased in a time-dependent manner, whereas those of patients 
Table 2 Summary of renal safety evaluation provided by included studies

\begin{tabular}{|c|c|c|c|}
\hline Study & Renal safety evaluation & Summary & \\
\hline \multirow{4}{*}{$\begin{array}{l}\text { Riveiro-Barciela } \\
\text { et al, } 2017^{15}\end{array}$} & Mean eGFR by MDRD, $\mathrm{mL} / \mathrm{min}$ & TDF-containing & BL: 90.8, I2th MO: 90.3, 36th MO: 88.9, 60th MO: 85.I \\
\hline & & ETV & BL: 8I.2, 12th MO: 79.0, 36th MO: 84.8, 60th MO: 90.7 \\
\hline & Mean creatinine, $\mathrm{mg} / \mathrm{dL}$ & TDF-containing & BL: 0.9, I2th MO: 0.9, 36th MO: 0.9I, 60th MO: 0.95 \\
\hline & & ETV & BL: 0.99, I2th MO: 1.0, 36th MO: 1.0, 60th MO: 0.9 \\
\hline \multirow[t]{6}{*}{$\begin{array}{l}\text { Koksal et al, } \\
2016^{16}\end{array}$} & $\begin{array}{l}\text { eGFR by CKD-EPI-CysC, mean } \\
\text { (SD), } \mathrm{mL} / \mathrm{min} / \mathrm{l} .73 \mathrm{~m}^{2}\end{array}$ & TDF & $\begin{array}{l}\text { BL: } 84.7 \text { (29.6), 3rd MO: } 82.8 \text { (45.9), 12th MO: } 79.7 \text { (36.3), 24th MO: } \\
76.9 \text { (30.8); } p=0.004\end{array}$ \\
\hline & & ETV & $\begin{array}{l}\text { BL: } 90.0 \text { (24.I), 3rd MO: } 96.6 \text { (81.6), I2th MO: } 92.9 \text { (43.3), 24th MO: } \\
84.5 \text { (29.5); } p=0.46\end{array}$ \\
\hline & $\begin{array}{l}\text { eGFR by CKD-EPI-Cr-CysC, } \\
\text { mean (SD), } \mathrm{mL} / \mathrm{min} / \mathrm{I} .73 \mathrm{~m}^{2}\end{array}$ & TDF & $\begin{array}{l}\text { BL: } 90.6 \text { (22.5), 3rd MO: } 82.7 \text { (3I.2), I2th MO: } 83.1 \text { (32.2), 24th MO: } \\
73.6 \text { (34.7); } p=0.05\end{array}$ \\
\hline & & ETV & $\begin{array}{l}\text { BL: } 93.5 \text { (19.6), 3rd MO: } 95.6 \text { (4I.I), I2th MO: } 88.7 \text { (3I.2), 24th MO: } \\
82.3 \text { (23.7); } p=0.17\end{array}$ \\
\hline & Creatinine, mean $(\mathrm{SD}), \mathrm{mg} / \mathrm{dL}$ & TDF & $\begin{array}{l}\text { BL: } 0.76(0.16), 3 \text { rd MO: } 0.86(0.19), \text { I } 2 \text { th MO: } 0.81 \text { (0.24), 24th MO: } \\
0.85(0.26) ; p=0.08\end{array}$ \\
\hline & & ETV & $\begin{array}{l}\text { BL: } 0.83(0.18), 3 r d \text { MO: } 0.84(0.14) \text {, I2th MO: } 0.82(0.13) \text {, 24th MO: } \\
0.80(0.16) ; p=0.16\end{array}$ \\
\hline \multirow{4}{*}{$\begin{array}{l}\text { López Centeno } \\
\text { et al, } 2016^{17}\end{array}$} & eGFR $<60 \mathrm{~mL} / \mathrm{min} / 1.73 \mathrm{~m}^{2}$ & TDF-containing & BL: 4 (12.5), I2th MO: 6 (19.4) \\
\hline & n (\%) & ETV & BL: 5 (I5.6), I2th MO: 5 (I5.6) \\
\hline & Creatinine $\geq 1.4$ mg/dL, $\mathrm{n}(\%)$ & TDF-containing & BL: 3 (9.4), 12th MO: 4 (12.9) \\
\hline & & ETV & BL: 2 (6.3), 12th MO: 2 (6.3) \\
\hline \multirow{15}{*}{$\begin{array}{l}\text { Rodríguez- } \\
\text { Nóvoa et al, } \\
2016^{3}\end{array}$} & Creatinine, median (SD), mg/dL & TDF & $0.95(0.15)$ \\
\hline & & ETV & $\mathrm{I} .02(0.20)$ \\
\hline & & Control & $0.93(0.16)$ \\
\hline & Phosphate, median (SD), mg/dL & TDF & $3.18(0.55)$ \\
\hline & & ETV & $3.19(0.54)$ \\
\hline & & Control & $3.28(0.45)$ \\
\hline & eGFR by MDRD4, median (SD), & TDF & $83(15)$ \\
\hline & $\mathrm{mL} / \mathrm{min}$ & ETV & $81(14)$ \\
\hline & & Control & $83(14)$ \\
\hline & Ratio protein/creatinine, & TDF & $80(43)$ \\
\hline & median (SD), mg/g & ETV & $66(59)$ \\
\hline & & Control & $63(28)$ \\
\hline & Ratio retinol-binding protein/ & TDF & $104(112)$ \\
\hline & creatinine, median (SD), $\mu \mathrm{g} / \mathrm{mL}$ & ETV & 71 (62) \\
\hline & & Control & $72(49)$ \\
\hline \multirow{8}{*}{$\begin{array}{l}\text { Zoulim et al, } \\
2016^{18}\end{array}$} & Creatinine increase from & TDF/ETV & 24th MO: I (I.I) \\
\hline & $\mathrm{BL} \geq 0.3 \mathrm{mg} / \mathrm{dL}, \mathrm{n}(\%)$ & & \\
\hline & Creatinine increase from & TDF/ETV & 24th MO: I (I.I) \\
\hline & $\mathrm{BL} \geq 0.5 \mathrm{mg} / \mathrm{dL}, \mathrm{n}(\%)$ & & \\
\hline & Creatinine clearance & TDF/ETV & 24th MO: I (I.I) \\
\hline & $<50$ mL/min, n (\%) & & \\
\hline & Phosphate $<2.0 \mathrm{mg} / \mathrm{dL}, \mathrm{n}(\%)$ & TDF/ETV & 24th MO: $2(2.2)$ \\
\hline & Phosphate $<2.3 \mathrm{mg} / \mathrm{dL}, \mathrm{n}(\%)$ & TDF/ETV & 24th MO: 8 (8.9) \\
\hline Sriprayoon & eGFR at BL, mean (SD), & TDF & $106.7(20.6)$ \\
\hline \multirow[t]{7}{*}{ et al, $2017^{19}$} & $\mathrm{~mL} / \mathrm{min} / 1.73 \mathrm{~m}^{2}$ & ETV & $105.3(22.3)$ \\
\hline & eGFR decrease $\geq 20 \%$, n (\%) & TDF & 12th MO: 18 (9.4), 24th MO: 33 (17.3), 36th MO: 32 (I6.8) \\
\hline & & ETV & I2th MO: 6 (3.I), 24th MO: I3 (6.7), 36th MO: 29 (14.9) \\
\hline & Phosphorus $<2.0 \mathrm{mg} / \mathrm{dL}, \mathrm{n}(\%)$ & TDF & I2th MO: $0(0.0), 24$ th MO: $0(0.0), 36$ th MO: $0(0.0)$ \\
\hline & & ETV & I2th MO: $0(0.0), 24$ th MO: $0(0.0), 36$ th MO: $0(0.0)$ \\
\hline & Fractional excretion of & TDF & 24th MO: 9.4 (3.7), 36th MO: 9.6 (3.8) \\
\hline & phosphate, mean (SD) & ETV & 24th MO: 8.9 (3.6), 36th MO: 8.7 (3.5) \\
\hline Tsai et al, & eGFR by MDRD, mean (SD), & TDF & Prior I2th MO: 80.1 (I3.3), BL: 78.3 (II.6), 24th MO: 73.0 (I3.I) \\
\hline \multirow[t]{5}{*}{$2016^{20}$} & $\mathrm{~mL} / \mathrm{min} / \mathrm{l} .73 \mathrm{~m}^{2}$ & ETV & Prior I2th MO: 76.2 (II.3), BL: 75.6 (9.5), 24th MO: 79.3 (I4.2) \\
\hline & Rise in CKD category with & TDF & $0(0.0)$ \\
\hline & $\geq 25 \%$ increase in eGFR, $n$ (\%) & ETV & $0(0.0)$ \\
\hline & Rise in CKD category with & TDF & I (2.7) \\
\hline & $<25 \%$ rise in eGFR, $n(\%)$ & ETV & I (I.6) \\
\hline
\end{tabular}


Table 2 (Continued)

\begin{tabular}{|c|c|c|c|}
\hline Study & Renal safety evaluation & Summary & \\
\hline & No change in CKD category, & TDF & $34(91.9)$ \\
\hline & n (\%) & ETV & $57(91.9)$ \\
\hline & Drop in CKD category with & TDF & I (2.7) \\
\hline & $<25 \%$ decrease in eGFR, $n(\%)$ & ETV & $4(6.5)$ \\
\hline & Drop in CKD category with & TDF & $\mathrm{I}(2.7)$ \\
\hline & $\geq 25 \%$ decrease in eGFR, $n(\%)$ & ETV & $0(0.0)$ \\
\hline \multirow[t]{3}{*}{$\begin{array}{l}\text { Park et al, } \\
2016^{21}\end{array}$} & $\begin{array}{l}\text { Creatinine, median (range), } \\
\mathrm{mg} / \mathrm{dL}\end{array}$ & TDF/ETV & BL: 0.87 (0.38-1.30), I2th MO: 0.90 (0.57-1.28) \\
\hline & Creatinine increase from & TDF/ETV & I (I.56) (BL: 0.60 mg/dL, 9th MO: I.36 mg/dL, I2th MO: 0.58 mg/dL) \\
\hline & $\mathrm{BL}>0.5 \mathrm{mg} / \mathrm{dL}, \mathrm{n}(\%)$ & & \\
\hline Wang et al, & eGFR, mean (SD), & TDF (TN) & BL: $98.9,6$ th MO: 94.5, I2th MO: $90.5,24$ th MO: $90.5,36$ th MO: 90.9 \\
\hline \multirow[t]{5}{*}{$2016^{22}$} & $\mathrm{~mL} / \mathrm{min} / \mathrm{l} .73 \mathrm{~m}^{2}$ & TDF (TE) & BL: $92.2,6$ th MO: 87.3, I2th MO: 86.7, 24th MO: 85.3, 36th MO: 84.3 \\
\hline & eGFR decrease $>20 \%, \mathrm{n}(\%)$ & TDF (TN, TE) & $38(16.89)$ \\
\hline & Creatinine increase from & TDF (TN, TE) & $8(3.56)$ \\
\hline & $\mathrm{BL} \geq 0.5 \mathrm{mg} / \mathrm{dL}, \mathrm{n}(\%)$ & & \\
\hline & Phosphate $<2.5 \mathrm{mg} / \mathrm{dL}, \mathrm{n}(\%)$ & TDF (TN, TE) & $14(8.28)$ \\
\hline \multirow{6}{*}{$\begin{array}{l}\text { Tsai et al, } \\
2016^{23}\end{array}$} & eGFR by MDRD, mean (SD), & TDF & BL: 92,12 th MO: $85.6,24$ th MO: 86.3 \\
\hline & $\mathrm{mL} / \mathrm{min} / \mathrm{l} .73 \mathrm{~m}^{2}$ & ETV & $\begin{array}{l}\text { BL: 86.I, I2th MO: 92.6, 24th MO: 94.4, 36th MO: 97, 48th MO: } \\
\text { I00.8, 60th MO: } 100.4\end{array}$ \\
\hline & & LdT & $\begin{array}{l}\text { BL: 8I.I, I2th MO: 8I.8, 24th MO: 79.3, 36th MO: 8I.I, 48th MO: } \\
84.7,60 \text { th MO: } 87.6\end{array}$ \\
\hline & Phosphate $<2.5$ mg/dL, $\mathrm{n}(\%)$ & TDF & $4(8.2)$ \\
\hline & & ETV & $\mathrm{I}(\mathrm{l} .8)$ \\
\hline & & LdT & I (5.3) \\
\hline \multirow[t]{9}{*}{$\begin{array}{l}\text { Koklu et al, } \\
2015^{24}\end{array}$} & $\begin{array}{l}\text { eGFR by MDRD, mean (SD), } \\
\mathrm{mL} / \mathrm{min} / \mathrm{l} .73 \mathrm{~m}^{2}\end{array}$ & TDF & $\begin{array}{l}\text { BL: I00.72 (25.19), Ist MO: } 96.44 \text { (24.27), 6th MO: } 97.13 \text { (23.95), } \\
\text { I2th MO: } 96.1 \text { ( } 24.42), 24 \text { th MO: } 96.72(25.67) ; p=0.00 \text { I }\end{array}$ \\
\hline & & ETV & $\begin{array}{l}\text { BL: } 96.20 \text { (22.53), Ist MO: } 96.37(21.01), 6 \text { th MO: } 95.87(21.84) \text {, } \\
\text { I2th MO: } 94.30 \text { (23.80), 24th MO: } 95.94(23.85) ; p=0.535\end{array}$ \\
\hline & & LAM & $\begin{array}{l}\text { BL: } 96.9 \text { I (25.I7), Ist MO: } 96.00 \text { (25.28), 6th MO: } 95.34(26.78) \text {, } \\
\text { I2th MO: } 97.50 \text { (25.39), 24th MO: } 96.23 \text { (24.07); } p=0.490\end{array}$ \\
\hline & Creatinine, mean (SD), mg/dL & TDF & $\begin{array}{l}\text { BL: } 0.85(0.42) \text {, Ist MO: } 0.90(0.56), 6 \text { th MO: } 0.89(0.5 \mathrm{I}) \text {, I 2th MO: } \\
0.89(0.42), 24 \text { th MO: } 0.90(0.58) ; p=0.00 \text { I }\end{array}$ \\
\hline & & ETV & $\begin{array}{l}\text { BL: } 0.86(0.19) \text {, I st MO: } 0.85(0.18), 6 \text { th MO: } 0.86(0.19) \text {, I2th MO: } \\
0.88(0.21), 24 \text { th MO: } 0.87(0.22) ; p=0.500\end{array}$ \\
\hline & & LAM & $\begin{array}{l}\text { BL: } 0.84(0.17) \text {, I st MO: } 0.85(0.18), 6 \text { th MO: } 0.88(0.42) \text {, I2th MO: } \\
0.84 \text { (0.19), 24th MO: } 0.85(0.21) ; p=0.11 \text { I }\end{array}$ \\
\hline & Phosphate, mean (SD), mg/dL & TDF & $\begin{array}{l}\text { BL: } 3.23(0.45) \text {, Ist MO: } 3.21 \text { ( } 0.49), 6 \text { th MO: } 3.21 \text { ( } 0.46) \text {, I2th MO: } \\
3.15(0.53), 24 \text { th MO: } 3.23(0.62) ; p=0.810\end{array}$ \\
\hline & & ETV & $\begin{array}{l}\text { BL: } 3.38 \text { (0.36), Ist MO: } 3.47(0.36), 6 \text { th MO: } 3.39(0.38) \text {, I2th MO: } \\
3.39(0.36), 24 \text { th MO: } 3.45(0.43) ; p=0.358\end{array}$ \\
\hline & & LAM & $\begin{array}{l}\text { BL: } 3.08(0.8 I) \text {, Ist MO: } 3.17(0.89), 6 \text { th MO: } 3.28(1.04) \text {, I2th MO: } \\
3.22(0.98), 24 \text { th MO: } 2.98(0.70) ; p=0.12 \text { I }\end{array}$ \\
\hline Kim et al, & Creatinine increase from & TDF/ETV & $0(0.0)$ \\
\hline \multirow[t]{11}{*}{$2015^{25}$} & $\mathrm{BL}>0.5 \mathrm{mg} / \mathrm{dL}, \mathrm{n}(\%)$ & TDF/LAM & $0(0.0)$ \\
\hline & Creatinine increase from & TDF/ETV & $5(18.52)$ \\
\hline & $\mathrm{BL}>0.3 \mathrm{mg} / \mathrm{dL}, \mathrm{n}(\%)$ & TDF/LAM & $5(20.00)$ \\
\hline & Creatinine clearance & TDF/ETV & $0(0.0)$ \\
\hline & $<50$ mL/min, $\mathrm{n}(\%)$ & TDF/LAM & $0(0.0)$ \\
\hline & Phosphate $<2.0 \mathrm{mg} / \mathrm{dL}, \mathrm{n}(\%)$ & TDF/ETV & $0(0.0)$ \\
\hline & & TDF/LAM & $0(0.0)$ \\
\hline & Phosphate $<2.7 \mathrm{mg} / \mathrm{dL}, \mathrm{n}(\%)$ & TDF/ETV & $2(7.4 I)$ \\
\hline & & TDF/LAM & $3(12.00)$ \\
\hline & $\begin{array}{l}\text { Change in creatinine, mean } \\
(\mathrm{IQR}), \mathrm{mg} / \mathrm{dL}\end{array}$ & TDF/ETV & $\begin{array}{l}\text { 6th MO: }-0.03 \text { ( }-0.25 \text { to } 0.23), 12 \text { th MO: } 0.11 \text { ( }(-0.1 \text { I to } 0.38) \text {, } \\
\text { I8th MO: } 0.21 \text { ( }-0.08 \text { to } 0.42)\end{array}$ \\
\hline & & TDF/LAM & $\begin{array}{l}\text { 6th MO: }-0.04(-0.21 \text { to } 0.24), 12 \text { th MO: } 0.08 \text { ( }-0.28 \text { to } 0.25) \text {, } \\
\text { I8th MO: } 0.19(-0.02 \text { to } 0.43)\end{array}$ \\
\hline
\end{tabular}


Table 2 (Continued)

\begin{tabular}{|c|c|c|c|}
\hline Study & Renal safety evaluation & Summary & \\
\hline & $\begin{array}{l}\text { Change in creatinine clearance, } \\
\text { mean (IQR), } \mathrm{mL} / \mathrm{min}\end{array}$ & TDF/ETV & $\begin{array}{l}\text { 6th MO: } 0.7 \text { ( }-60.9 \text { to 16.9), I2th MO: }-12.5 \text { ( }-63.2 \text { to } 7.3) \text {, } \\
\text { I8th MO: }-22.1 \text { ( }-64.3 \text { to } 6.9)\end{array}$ \\
\hline & & TDF/LAM & $\begin{array}{l}\text { 6th MO: } 2.4 \text { ( }-26.5 \text { to } 16.4), \text { I2th MO: }-7.5 \text { ( }-31.9 \text { to } 33.4) \text {, } \\
\text { I8th MO: }-21.3(-37.0 \text { to }-8.9)\end{array}$ \\
\hline \multirow[t]{4}{*}{ Ha et al, $2015^{26}$} & Patients reclassified to a higher & TDF & $16(15.5)$ \\
\hline & $\begin{array}{l}\text { category of renal impairment } \\
\text { classification, } \mathrm{n}(\%)\end{array}$ & ETV & $18(17.5)$ \\
\hline & $\begin{array}{l}\% \text { change in eGFR from } B L \text { in } \\
\text { patients reclassified to a more }\end{array}$ & TDF & $\begin{array}{l}<10 \%: 3(19), 10 \%-19.99 \%: 4 \text { (25), 20\%-29.99\%: } 7 \text { (44), 30\%-39.99\%: } \\
2 \text { (13), >40\%: } 0 \text { (0) }\end{array}$ \\
\hline & $\begin{array}{l}\text { severe renal classification on } \\
\text { treatment, } \mathrm{n}(\%)\end{array}$ & ETV & $\begin{array}{l}<10 \%: 2(11), 10 \%-19.99 \%: 9 \text { (50), 20\%-29.99\%: } 6 \text { (33), 30\%-39.99\%: } \\
0 \text { (0), >40\%: I (6) }\end{array}$ \\
\hline \multirow{8}{*}{$\begin{array}{l}\text { Lim et al, } \\
2016^{27}\end{array}$} & Creatinine increase from & $\mathrm{TDF} \rightarrow \mathrm{TDF}$ & $0(0.0)$ \\
\hline & $\mathrm{BL} \geq 0.5 \mathrm{mg} / \mathrm{dL}, \mathrm{n}(\%)$ & $\begin{array}{l}\text { TDF/ } \\
\text { ETV } \rightarrow \text { TDF }\end{array}$ & $0(0.0)$ \\
\hline & eGFR $<50 \mathrm{~mL} / \mathrm{min} / 1.73 \mathrm{~m}^{2}$ & $\mathrm{TDF} \rightarrow \mathrm{TDF}$ & $0(0.0)$ \\
\hline & n (\%) & TDF/ & $0(0.0)$ \\
\hline & & $\mathrm{ETV} \rightarrow \mathrm{TDF}$ & \\
\hline & Phosphate $<2.0 \mathrm{mg} / \mathrm{dL}, \mathrm{n}(\%)$ & $\mathrm{TDF} \rightarrow \mathrm{TDF}$ & $0(0.0)$ \\
\hline & & TDF/ & $0(0.0)$ \\
\hline & & $\mathrm{ETV} \rightarrow \mathrm{TDF}$ & \\
\hline \multirow{4}{*}{$\begin{array}{l}\text { Hung et al, } \\
2015^{28}\end{array}$} & Creatinine increase from & TDF & $2(6.67)$ \\
\hline & $\mathrm{BL} \geq 0.5 \mathrm{mg} / \mathrm{dL}$ at $6 \mathrm{MO}, \mathrm{n}(\%)$ & ETV & $2(2.02)$ \\
\hline & Mean eGFR by MDRD, & TDF & BL: 108,6 th MO: $87 ; p=0.001$ \\
\hline & $\mathrm{mL} / \mathrm{min} / \mathrm{l} .73 \mathrm{~m}^{2}$ & ETV & BL: 92,6 th MO: $84 ; p=0.00 \mathrm{I}$ \\
\hline \multirow{6}{*}{$\begin{array}{l}\text { Lim et al, } \\
2016^{29}\end{array}$} & Creatinine increase from & TDF & $0(0.0)$ \\
\hline & $\mathrm{BL} \geq 0.5$ mg/dL, n (\%) & TDF/ETV & $0(0.0)$ \\
\hline & eGFR $<50 \mathrm{~mL} / \mathrm{min} / 1.73 \mathrm{~m}^{2}$ & TDF & I (2.22) \\
\hline & n (\%) & TDF/ETV & $0(0.0)$ \\
\hline & Phosphate $<2.0$ mg/dL, n (\%) & TDF & $0(0.0)$ \\
\hline & & TDF/ETV & I (2.22) \\
\hline \multirow[t]{15}{*}{ Qi et al, 2015 30} & Change in creatinine, $\mathrm{mg} / \mathrm{dL}$ & Untreated & I2th MO: $0.004,24$ th MO: $0.012,36$ th MO: 0.030 \\
\hline & & LAM & I2th MO: $0.018,24$ th MO: $0.047,36$ th MO: 0.082 \\
\hline & & ADV & I2th MO: 0.07 I, 24th MO: $0.128,36$ th MO: 0.314 \\
\hline & & LdT & 12th MO: $-0.066,24$ th MO: $-0.100,36$ th MO: -0.135 \\
\hline & & ETV & I2th MO: $0.006,24$ th MO: $-0.023,36$ th MO: 0.007 \\
\hline & Change in eGFR by CG, & Untreated & I2th MO: $-0.665,24$ th MO: $-0.892,36$ th MO: -1.047 \\
\hline & $\mathrm{mL} / \mathrm{min}$ & LAM & I2th MO: $-4.530,24$ th MO: $-8.817,36$ th MO: -11.637 \\
\hline & & ADV & I2th MO: $-5.623,24$ th MO: $-11.260,36$ th MO: -13.720 \\
\hline & & LdT & 12th MO: 8.232, 24th MO: 14.998, 36th MO: 23.619 \\
\hline & & ETV & I2th MO: $-0.001,24$ th MO: I.806, 36th MO: -1.358 \\
\hline & Change in eGFR by MDRD, & Untreated & I2th MO: $-0.692,24$ th MO: $-1.07 \mathrm{I}, 36$ th MO: -1.799 \\
\hline & $\mathrm{mL} / \mathrm{min}$ & LAM & I2th MO: $-4.715,24$ th MO: -10.166 , 36th MO: -12.410 \\
\hline & & ADV & I2th MO: -6.922 , 24th MO: $-11.637,36$ th MO: -15.38 I \\
\hline & & LdT & I2th MO: 9.570, 24th MO: 15.428, 36th MO: 26.236 \\
\hline & & ETV & I2th MO: $-0.002,24$ th MO: $1.988,36$ th MO: -1.284 \\
\hline \multirow{9}{*}{$\begin{array}{l}\text { Tien et al, } \\
2015^{31}\end{array}$} & eGFR by CG, mean (SD), & Untreated & $118(36)$ \\
\hline & $\mathrm{mL} / \mathrm{min}$ & TDF & $108(29)$ \\
\hline & & ETV & $103(26)$ \\
\hline & eGFR by MDRD, mean (SD), & Untreated & II 8 (28) \\
\hline & $\mathrm{mL} / \mathrm{min}$ & TDF & $103(26)$ \\
\hline & & ETV & $102(22)$ \\
\hline & Phosphate, mean (SD), mg/dL & Untreated & $3.4(0.5)$ \\
\hline & & TDF & $3.4(0.5)$ \\
\hline & & ETV & $3.5(0.5)$ \\
\hline
\end{tabular}


Table 2 (Continued)

\begin{tabular}{|c|c|c|c|}
\hline \multirow[t]{2}{*}{ Study } & \multirow{2}{*}{$\begin{array}{l}\text { Renal safety evaluation } \\
\text { Phosphate }<2.8 \mathrm{mg} / \mathrm{dL}, \mathrm{n}(\%)\end{array}$} & \multicolumn{2}{|l|}{ Summary } \\
\hline & & Untreated & $5(8)$ \\
\hline & & TDF & $6(14)$ \\
\hline & & ETV & $2(4)$ \\
\hline & Creatinine, mean (SD), mg/dL & Untreated & $0.65(0.17)$ \\
\hline & & TDF & $0.79(0.22)$ \\
\hline & & ETV & $0.76(0.19)$ \\
\hline & Creatinine $>1.5 \mathrm{mg} / \mathrm{dL}, \mathrm{n}(\%)$ & Untreated & $0(0)$ \\
\hline & & TDF & $0(0)$ \\
\hline & & ETV & $0(0)$ \\
\hline \multirow{4}{*}{$\begin{array}{l}\text { Lok et al, } \\
2012^{32}\end{array}$} & Creatinine increase from & TDF/ETV & $4(2.0)$ \\
\hline & $\mathrm{BL} \geq 0.3 \mathrm{mg} / \mathrm{dL}, \mathrm{n}(\%)$ & ETV & $6(3.3)$ \\
\hline & Creatinine increase from & TDF/ETV & $0(0.0)$ \\
\hline & $\mathrm{BL} \geq 0.5 \mathrm{mg} / \mathrm{dL}, \mathrm{n}(\%)$ & ETV & $3(1.6)$ \\
\hline \multirow{7}{*}{$\begin{array}{l}\text { Gish et al, } \\
2012^{33}\end{array}$} & Creatinine increase from & TDF & $22(27.5)$ \\
\hline & $\mathrm{BL} \geq 0.2 \mathrm{mg} / \mathrm{dL}, \mathrm{n}(\%)$ & ETV & $23(28.8)$ \\
\hline & Creatinine increase from & TDF & $3(3.8)$ \\
\hline & $\mathrm{BL} \geq 0.5 \mathrm{mg} / \mathrm{dL}, \mathrm{n}(\%)$ & ETV & II (I3.8) \\
\hline & eGFR decrease $\geq 20 \%$ (CG), & TDF & $28(35.0)$ \\
\hline & $\mathrm{n}(\%)$ & ETV & $29(36.3)$ \\
\hline & eGFR decrease $\geq 20 \%$ (MDRD), & $\begin{array}{l}\text { TDF } \\
\text { ETV }\end{array}$ & $\begin{array}{l}33(41.3) \\
35(43.8)\end{array}$ \\
\hline \multirow{6}{*}{$\begin{array}{l}\text { Liaw et al, } \\
201 I^{34}\end{array}$} & Creatinine increase from & TDF & $4(8.9)$ \\
\hline & $\mathrm{BL} \geq 0.5$ mg/dL, n (\%) & TDF/FTC & $\mathrm{I}(2.2)$ \\
\hline & & ETV & I (4.5) \\
\hline & Phosphate $<2.0 \mathrm{mg} / \mathrm{dL}, \mathrm{n}(\%)$ & TDF & I (2.2) \\
\hline & & TDF/FTC & $2(4.4)$ \\
\hline & & ETV & $0(0.0)$ \\
\hline
\end{tabular}

Notes: The change in eGFR was calculated by ( 2 nd year eGFR - baseline eGFR)/baseline eGFR $\times 100 \%$. Categories of CKD were defined based on eGFR: $\geq 90,60-89,59-30$, and $<30 \mathrm{~mL} / \mathrm{min} / 1.73 \mathrm{~m}^{2}$, respectively. Classification of eGFR is as follows: unimpaired (eGFR $>80 \mathrm{~mL} / \mathrm{min}$ ), mildly impaired (50 $\left.\mathrm{mL} / \mathrm{min} \leq \mathrm{eGFR} \leq 80 \mathrm{~mL} / \mathrm{min}\right), \mathrm{moderately}$ impaired $(30 \mathrm{~mL} / \mathrm{min} \leq \mathrm{eGFR}<50 \mathrm{~mL} / \mathrm{min})$, and severely impaired (eGFR $<30 \mathrm{~mL} / \mathrm{min}$ ).

Abbreviations: BL, baseline; CKD-EPI-CysC, Chronic Kidney Disease Epidemiology Collaboration and cystatin C; CKD-EPI-Cr-CysC, Chronic Kidney Disease Epidemiology Collaboration and creatinine plus cystatin C; CG, Cockcroft-Gault; eGFR, estimated glomerular filtration rate; ETV, entecavir; FTC, emtricitabine; IQR, interquartile range; LAM, lamivudine; LdT, telbivudine; MDRD, modification of diet in renal disease; MDRD4, modification of diet in renal disease 4-variable version; MO, month; SD, standard deviation; TDF, tenofovir disoproxil fumarate; TN, treatment-naïve; TE, treatment-experienced.

treated with ETV increased or decreased across various time points. ${ }^{15,16,20,23,24,28}$ Similar percentage of patients in both the treatment groups showed $\geq 20 \%$ decrease in eGFR during the treatment (based on CG, TDF 35.0\% vs ETV 36.3\%; based on MDRD, TDF 41.3\% vs ETV 43.8\%). ${ }^{33}$ A similar tendency was also observed in a recent clinical trial conducted in Thailand. ${ }^{19}$ After 36 months, $16.8 \%$ and $14.9 \%$ of patients receiving TDF and ETV, respectively, experienced $\geq 20 \%$ decrease in eGFR; however, the decrease was observed in more patients receiving TDF than in those receiving ETV at 12 and 24 months. ${ }^{19}$ Around $30 \%$ of patients in both TDF and ETV groups experienced $\mathrm{a} \geq 0.2 \mathrm{mg} / \mathrm{dL}$ increase in creatinine from BL; however, creatinine increase of $\geq 0.5 \mathrm{mg} / \mathrm{dL}$ from $\mathrm{BL}$ occurred in more patients receiving ETV than in those receiving TDF $(13.8 \%$ vs $3.8 \%$; $p=0.025) .{ }^{33}$ The frequencies of creatinine elevation by $\geq 0.3 \mathrm{mg} / \mathrm{dL}$ were similar in both groups (TDF/ETV 2.0\% vs ETV 3.3\%); however, creatinine elevation by $\geq 0.5 \mathrm{mg} / \mathrm{dL}$ was more frequent in patients treated with ETV alone (TDF/ETV 0.0\% vs ETV 1.6\%). ${ }^{32}$
These heterogeneous results may be partially attributed to different characteristics, such as comorbidities and coadministered drugs, of the study subjects.

According to multivariate analyses, various risk factors, such as advanced age, preexisting renal failure, comorbidities, history of transplant, concomitant nephrotoxic drugs, advanced HIV coinfection, and male gender, were associated with eGFR reductions by TDF or ETV. ${ }^{23,26,33,35,36}$ Especially, preexisting renal insufficiency was a major independent risk factor for deterioration of renal function during the treatment of chronic HBV infection. ${ }^{23,26,33}$ Moreover, previous studies have reported an association of $\mathrm{CHB}$ with CKD, and $\sim 15 \%-30 \%$ of patients with CHB showed $\mathrm{BL}$ renal insufficiency or comorbidities that were likely to cause CKD, such as diabetes mellitus (DM) and HTN. ${ }^{7-11}$ However, TDF therapy was not significantly associated with changes in renal function when compared with ETV therapy. ${ }^{26,33}$ Large proportions of TDF and ETV are also renally excreted in their unchanged forms. ${ }^{37,38}$ Thus, NUCs 
other than TDF and ETV may be considered to prevent the progression of renal decline in patients with $\mathrm{CHB}$ and decreased renal functions.

Compared with TDF, tenofovir alafenamide (TAF), a novel prodrug of tenofovir, led to approximately four times higher intracellular concentrations of tenofovir diphosphate, an active metabolite, which may result in much lower doses of TAF than those of TDF. ${ }^{39,40}$ Consequently, 90\% lower systemic exposure of tenofovir was expected in patients treated with TAF than in those treated with TDF. ${ }^{40}$ This is likely to reduce the risk for tenofovir-associated renal toxicity. According to a clinical trial conducted in patients infected with HIV-1, decreases or slight increases from BL to Week 48 in total urinary protein, albumin, retinol-binding protein, and $\beta_{2}$-microglobulin to urine creatinine ratios were observed in the TAF group; however, increases from BL to Week 48 in the protein to urine creatinine ratios were reported in the TDF group..$^{40}$ Two recent randomized clinical trials conducted in patients with $\mathrm{HBeAg-negative} \mathrm{or} \mathrm{-positive} \mathrm{chronic}$ HBV infection reported that TAF not only was non-inferior to TDF but also improved the negative effect of tenofovir on renal function. ${ }^{41,42}$ In patients with $\mathrm{HBeAg}$-negative chronic $\mathrm{HBV}$ infection, a small mean increase in creatinine from BL to Week 48 was reported in both TAF and TDF groups, and at Week 48, a median decrease in eGFR by CG was lower in patients treated with TAF than in those treated with TDF. ${ }^{41}$ Significantly smaller increases from BL to Week 48 in the markers of proximal tubular dysfunction, retinol-binding protein, and $\beta_{2}$-microglobulin to urine creatinine ratios were noted in the TAF group than in the TDF group. ${ }^{41}$ Similar tendencies were observed in patients with $\mathrm{HBeAg}$-positive chronic HBV infection. ${ }^{42}$ A network meta-analysis conducted by Chan et $\mathrm{al}^{7}$ reported that telbivudine (LdT) consistently improved renal functions measured by eGFR independent of measuring methods. In particular, tenofovir monotherapy caused decreases in eGFR, but combinational therapy of tenofovir with LdT improved renal functions. ${ }^{7}$

According to the WHO guidelines for the treatment of CHB in 2015, measuring BL renal function and assessing $\mathrm{BL}$ risks for renal dysfunction are recommended before commencing antiviral therapy. ${ }^{43}$ In cases where BL patients have eGFR $<50 \mathrm{~mL} / \mathrm{min}$ or risk factors for renal insufficiency, such as long-term DM, uncontrolled HTN, and severe bone-related diseases, tenofovir should be avoided, its dose should be adjusted, or ETV should be used. ${ }^{43}$ Thus, as shown in previous randomized clinical studies, ${ }^{40,41}$ TAF could be considered as the first drug of choice for the treatment of $\mathrm{CHB}$ in patients with reduced renal function or in those with risk factors for renal dysfunction. In addition, LdT monotherapy or combinational therapy with TAF could be another option for these patients; however, well-organized, randomized clinical trials are necessary to prove renal safety when TAF + LdT or LdT alone is administered to these patients.

This study had some limitations that should be addressed. Two electronic databases (ie, PubMed and ClinicalTrials.gov website) were utilized to search relevant clinical trials, although various databases are available. This limited database utilization also likely limited our opportunities to search additional valuable and relevant clinical trials. Almost all of the selected clinical trials mentioned that TDF and ETV were not likely to have significantly negative effects on renal functions. However, consistent results were not shown partially owing to the different characteristics of study subjects and various markers used to measure renal functions, which made the conducting of further meta-analysis difficult.

\section{Conclusion}

The data reported in this study suggest that use of TDF and ETV could be associated with reductions in kidney function in patients with chronic HBV infection. The eGFRs of patients treated with TDF were reduced in a time-dependent manner, whereas the eGFRs of patients treated with ETV increased or decreased across various time points. TAF as the first drug of choice for the treatment of chronic HBV infection could be used in patients with decreased renal function or in those with risk factors for renal dysfunction, and $\mathrm{TAF}+\mathrm{LdT}$ or LdT alone could also be considered for these patients. However, well-organized, prospective, large-scale, randomized clinical trials are necessary to determine the renal safety of TAF + LdT or LdT alone for the treatment of such patients. In addition, studies on management strategies for $\mathrm{HBV}$-infected patients with various risk factors (eg, advanced age, pre-existing renal failure, comorbidities, history of transplant, and concomitant nephrotoxic drugs) associated with reduction in eGFR are warranted in the near future.

\section{Acknowledgment}

This work was supported by the National Research Foundation of Korea (NRF) grant funded by the Korea government (No 2016R1C1B1015938).

\section{Disclosure}

The authors report no conflicts of interest in this work.

\section{References}

1. WHO [webpage on the Internet]. WHO Fact Sheet on Hepatitis B. 2016. Available from: http://www.who.int/mediacentre/factsheets/fs204/en/. Accessed March 14, 2017. 
2. Shin JH, Kwon HJ, Jang HR, et al. Risk factors for renal functional decline in chronic hepatitis B patients receiving oral antiviral agents. Medicine (Baltimore). 2016;95(1):e2400.

3. Rodríguez-Nóvoa S, García-Samaniego J, Prieto M, et al. Altered underlying renal tubular function in patients with chronic hepatitis B receiving nucleos(t)ide analogs in a real-world setting: the MENTE study. J Clin Gastroenterol. 2016;50(9):779-789.

4. Pol S, Lampertico P. First-line treatment of chronic hepatitis B with entecavir or tenofovir in 'real-life' settings: from clinical trials to clinical practice. J Viral Hepat. 2012;19(6):377-386.

5. Wu X, Cai S, Li Z, et al. Potential effects of telbivudine and entecavir on renal function: a systematic review and meta-analysis. Virol J. 2016; 13:64.

6. Lampertico P, Chan HL, Janssen HL, Strasser SI, Schindler R, Berg T. Review article: long-term safety of nucleoside and nucleotide analogues in HBV-monoinfected patients. Aliment Pharmacol Ther. 2016; 44(1):16-34.

7. Chan HL, Shaikh J, Gupta S, Hamed K. Renal function in nucleos(t) ide analog-treated patients with chronic hepatitis B: a systematic literature review and network meta-analysis. Adv Ther. 2016;33(5): $862-875$.

8. Chacko EC, Surrun SK, Mubarack Sani TP, Pappachan JM. Chronic viral hepatitis and chronic kidney disease. Postgrad Med J. 2010; 86(1018):486-492.

9. Bhimma R, Coovadia HM. Hepatitis B virus-associated nephropathy. Am J Nephrol. 2004;24(2):198-211.

10. Yi Z, Jie YW, Nan Z. The efficacy of anti-viral therapy on hepatitis B virus-associated glomerulonephritis: a systematic review and metaanalysis. Ann Hepatol. 2011;10(2):165-173.

11. Fabrizi F, Dixit V, Martin P. Meta-analysis: anti-viral therapy of hepatitis B virus-associated glomerulonephritis. Aliment Pharmacol Ther. 2006;24(5):781-788.

12. Lai KN, Li PK, Lui SF, et al. Membranous nephropathy related to hepatitis B virus in adults. N Engl J Med. 1991;324(21):1457-1463.

13. Appel G. Viral infections and the kidney: HIV, hepatitis B, and hepatitis C. Cleve Clin J Med. 2007;74(5):353-360.

14. Fontana RJ. Side effects of long-term oral antiviral therapy for hepatitis B. Hepatology. 2009;49(5 suppl):S185-S195.

15. Riveiro-Barciela M, Tabernero D, Calleja JL, et al. Effectiveness and safety of entecavir or tenofovir in a Spanish cohort of chronic hepatitis B patients: validation of the page-G score to predict hepatocellular carcinoma. Dig Dis Sci. 2017;62(3):784-793.

16. Koksal AR, Alkim H, Boga S, et al. Value of cystatin C-based e-GFR measurements to predict long-term tenofovir nephrotoxicity in patients with hepatitis B. Am J Ther. Epub 2016 Oct 4.

17. López Centeno B, Collado Borrell R, Pérez Encinas M, Gutiérrez García ML, Sanmartin Fenollera P. Comparison of the effectiveness and renal safety of tenofovir versus entecavir in patients with chronic hepatitis B. Farm Hosp. 2016;40(4):279-286.

18. Zoulim F, Białkowska-Warzecha J, Diculescu MM, et al. Entecavir plus tenofovir combination therapy for chronic hepatitis B in patients with previous nucleos(t)ide treatment failure. Hepatol Int. 2016;10(5): 779-788.

19. Sriprayoon T, Mahidol C, Ungtrakul T, et al. Efficacy and safety of entecavir versus tenofovir treatment in chronic hepatitis B patients: a randomized controlled trial. Hepatol Res. 2017;47(3):E161-E168.

20. Tsai MC, Chen $\mathrm{CH}$, Tseng PL, et al. Does nucleos(t)ide analogues treatment affect renal function in chronic hepatitis B patients who have already decreased eGFR? A longitudinal study. PLoS One. 2016;11(3): e0149761.

21. Park JY, Kim CW, Bae SH, et al. Entecavir plus tenofovir combination therapy in patients with multidrug-resistant chronic hepatitis B: results of a multicentre, prospective study. Liver Int. 2016;36(8):1108-1115.

22. Wang HM, Hung CH, Lee CM, et al. Three-year efficacy and safety of tenofovir in nucleos(t)ide analog-naïve and nucleos(t)ide analogexperienced chronic hepatitis B patients. J Gastroenterol Hepatol. 2016; 31(7):1307-1314.
23. Tsai MC, Chen $\mathrm{CH}$, Tseng PL, et al. Comparison of renal safety and efficacy of telbivudine, entecavir and tenofovir treatment in chronic hepatitis B patients: real world experience. Clin Microbiol Infect. 2016; 22(1):95.e1-e7.

24. Koklu S, Gulsen MT, Tuna Y, et al. Differences in nephrotoxicity risk and renal effects among anti-viral therapies against hepatitis B. Aliment Pharmacol Ther. 2015;41(3):310-319.

25. Kim BG, Jung SW, Kim EH, et al. Tenofovir-based rescue therapy for chronic hepatitis B patients who had failed treatment with lamivudine, adefovir, and entecavir. J Gastroenterol Hepatol. 2015;30(10): 1514-1521.

26. Ha NB, Ku K, Ha NB, Chaung KT, Trinh HN, Nguyen MH. Renal function in chronic hepatitis B patients treated with tenofovir disoproxil fumarate or entecavir monotherapy: a matched case-cohort study. J Clin Gastroenterol. 2015;49(10):873-877.

27. Lim YS, Yoo BC, Byun KS, et al. Tenofovir monotherapy versus tenofovir and entecavir combination therapy in adefovir-resistant chronic hepatitis B patients with multiple drug failure: results of a randomised trial. Gut. 2016;65(6):1042-1051

28. Hung CH, Hu TH, Lu SN, et al. Tenofovir versus entecavir in treatment of chronic hepatitis B virus with severe acute exacerbation. Antimicrob Agents Chemother. 2015;59(6):3168-3173.

29. Lim YS, Byun KS, Yoo BC, et al. Tenofovir monotherapy versus tenofovir and entecavir combination therapy in patients with entecavirresistant chronic hepatitis B with multiple drug failure: results of a randomised trial. Gut. 2016;65(5):852-860

30. Qi X, Wang JY, Mao RC, Zhang JM. Impact of nucleos(t)ide analogues on the estimated glomerular filtration rate in patients with chronic hepatitis B: a prospective cohort study in China. J Viral Hepat. 2015; 22(1):46-54.

31. Tien $\mathrm{C}, \mathrm{Xu}$ JJ, Chan LS, et al. Long-term treatment with tenofovir in Asian-American chronic hepatitis B patients is associated with abnormal renal phosphate handling. Dig Dis Sci. 2015;60(2):566-572.

32. Lok AS, Trinh H, Carosi G, et al. Efficacy of entecavir with or without tenofovir disoproxil fumarate for nucleos(t)ide-naïve patients with chronic hepatitis B. Gastroenterology. 2012;143(3):619-628.

33. Gish RG, Clark MD, Kane SD, Shaw RE, Mangahas MF, Baqai S. Similar risk of renal events among patients treated with tenofovir or entecavir for chronic hepatitis B. Clin Gastroenterol Hepatol. 2012; 10(8):941-946.

34. Liaw YF, Sheen IS, Lee CM, et al. Tenofovir disoproxil fumarate (TDF), emtricitabine/TDF, and entecavir in patients with decompensated chronic hepatitis B liver disease. Hepatology. 2011;53(1):62-72.

35. Fernandez-Fernandez B, Montoya-Ferrer A, Sanz AB, et al. Tenofovir nephrotoxicity: 2011 update. AIDS Res Treat. 2011;2011:354908.

36. Nelson MR, Katlama C, Montaner JS, et al. The safety of tenofovir disoproxil fumarate for the treatment of HIV infection in adults: the first 4 years. AIDS. 2007;21:1273-1281.

37. Product Information: VIREAD(R) oral tablets, tenofovir disoproxil fumarate oral tablets. Foster City, CA: Gilead Sciences, Inc.; 2010.

38. Product Information: BARACLUDE(R) oral tablets, solution, entecavir oral tablets, solution. Princeton, NJ: Bristol-Myers Squibb Company; 2009.

39. Ruane PJ, DeJesus E, Berger D, et al. Antiviral activity, safety, and pharmacokinetics/pharmacodynamics of tenofovir alafenamide as 10-day monotherapy in HIV-1-positive adults. J Acquir Immune Defic Syndr. 2013;63(4):449-455.

40. Sax PE, Wohl D, Yin MT, et al. Tenofovir alafenamide versus tenofovir disoproxil fumarate, coformulated with elvitegravir, cobicistat, and emtricitabine, for initial treatment of HIV-1 infection: two randomised, double-blind, phase 3, non-inferiority trials. Lancet. 2015;385(9987): 2606-2615.

41. Buti M, Gane E, Seto WK, et al. Tenofovir alafenamide versus tenofovir disoproxil fumarate for the treatment of patients with $\mathrm{HBeAg}$ negative chronic hepatitis B virus infection: a randomised, double-blind, phase 3, non-inferiority trial. Lancet Gastroenterol Hepatol. 2016;1(3): 196-206. 
42. Chan HL, Fung S, Seto WK, et al. Tenofovir alafenamide versus tenofovir disoproxil fumarate for the treatment of $\mathrm{HBeAg}$-positive chronic hepatitis B virus infection: a randomised, double-blind, phase 3, noninferiority trial. Lancet Gastroenterol Hepatol. 2016;1(3):185-195.
43. WHO [webpage on the Internet]. WHO Guidelines for the Prevention, Care and Treatment of Persons with Chronic Hepatitis B Infection. 2015. Available from: http://www.who.int/hepatitis/publications/ hepatitis-b-guidelines/en/. Accessed May 30, 2017.

\section{Publish your work in this journal}

Therapeutics and Clinical Risk Management is an international, peerreviewed journal of clinical therapeutics and risk management, focusing on concise rapid reporting of clinical studies in all therapeutic areas outcomes, safety, and programs for the effective, safe, and sustained use of medicines. This journal is indexed on PubMed Central, CAS
EMBase, Scopus and the Elsevier Bibliographic databases. The manuscript management system is completely online and includes a very quick and fair peer-review system, which is all easy to use. Visit http://www.dovepress.com/testimonials.php to read real quotes from published authors.

Submit your manuscript here: http://www.dovepress.com/therapeutics-and-clinical-risk-management-journal 